\title{
LEVEL CROSSING RATE OF MACRODIVERSITY OUTPUT PROCESS IN THE PRESENCE OF $\eta-\mu$ SHORT TERM FADING AND GAMMA LONG TERM FADING
}

\author{
UDC 621.391:621.395.38
}

\section{Srđan Milosavljević ${ }^{1}$, Dejan Milić ${ }^{2}$, Slaviša Trajković ${ }^{1}$, Petar Spalević ${ }^{3}$, Bojana Milosavljević ${ }^{4}$}

${ }^{1}$ University of Priština, Faculty of Economics, Department of Mathematics, Informatics and Statistics, Kosovska Mitrovica, Republic of Serbia

${ }^{2}$ University of Niš, Faculty of Electronic Engineering, Department of

Telecommunication, Niš, Republic of Serbia

${ }^{3}$ Faculty of Technical Science, Kosovska Mitrovica, Republic of Serbia

${ }^{4}$ Higher Technical Professional School in Zvečan, Zvečan, Republic of Serbia

\begin{abstract}
In this paper macrodiversity reception with macrodiversity selection combining (SC) receiver and two microdiversity MRC receivers operating over shadowed multipath fading channel is studied. Received signal experiences $\eta-\mu$ short term fading and correlated Gamma long term fading resulting in system performance degradation. Level crossing rate (LCR) of $\eta-\mu$ random process and level crossing rate of signals at outputs of microdiversity MRC receivers are efficiently calculated. By using these derived formulas, level crossing rate of macrodiversity $S C$ receiver output signal process is calculated. By using this result, average fade duration (AFD) of the proposed wireless communication system can be calculated. The influence of $\eta-\mu$ short term fading severity parameter, Gamma long term fading severity parameter and Gamma long term correlation coefficient on level crossing rate is analysed and studied.
\end{abstract}

Key words: Gamma shadowing, level crossing rate (LCR), $\eta$ - $\mu$ fading, macrodiversity system, microdiversity system, wireless communication system.

\section{INTRODUCTION}

Long term fading and short term fading degrade level crossing rate, average fade duration and outage probability of wireless mobile communication radio system. Macrodiversity technique can be used to reduce short term fading effects and long term

Received April 25, 2017

Corresponding author: Srđan Milosavljević

University of Pristina, Faculty of Economics, 38220 Kosovska Mitrovica, Republic of Serbia

E-mail: srdjan.milosavljevic@pr.ac.rs 
fading effects on bit error probability, outage probability, system capacity and average fade duration of wireless radio system simultaneously. Macrodiversity receiver combines signals with antennas at base stations resulting in long term fading effects reduction and microdiversity MRC (maximal ratio combining) combines signal envelopes with multiple antennas at base station resulting in short term fading effects reduction. Macrodiversity $\mathrm{SC}$ receiver selects microdiversity MRC receiver with the highest signal envelope average power of inputs. The square of signal at output of microdiversity MRC receiver is equal to the sum of square signal envelopes at its inputs.

The $\eta-\mu$ distribution can describe small scale signal envelope variation in non line of sight multipath fading channels when in phase component power and quadrature component power have different values. This distribution has two parameters. The parameter $\mu$ is severity parameter and $\eta$ is ratio of in phase component power and quadrature component power. The $\eta-\mu$ distribution is general distribution and Nakagami$\mathrm{m}$, Nakagami- $\mathrm{q}$ and Rayleigh distribution can be derived from $\eta-\mu$ distribution. For $\eta=1$, the $\eta-\mu$ distribution reduce to Nakagami-m distribution with $m=\mu$, for $\eta=0$, Nakagami$m$ distribution is also derived from $\eta-\mu$ distribution with $m=\mu / 2$, for $\mu=1$, the $\eta-\mu$ distribution reduces to Nakagami-q distribution and for $\eta=1$ and $\mu=1$, Raiyleigh distribution is obtained from $\eta-\mu$ distribution. When $\mu$ goes to infinity, $\eta-\mu$ short term fading channel becomes no fading channel [1], [2], [3].

The level crossing rate (LCR) is the second order performance measure of wireless radio communication system defined as the number of crossings at determined level in positive or negative direction and can be evaluated as average value of the first derivative of random process [4]. Average fade duration is, also, the second order performance measure defined as the probability that signal envelope falls below a specified threshold and can be evaluated as ratio of outage probability and level crossing rate [5]. The outage probability is the first order performance measure and can be evaluated from cumulative distribution [6].

There are more works in available technical literature considering level crossing rate of macrodiversity output signal process in the of presence long term fading and short term fading [7] - [15]. In paper [7], macrodiversity system with macrodiversity SC receiver and two microdiversity MRC receivers operating over Gamma shadowed Nakagami-m multipath fading channel are considered. The level crossing rate and average fade duration of the proposed system are evaluated. The second order statistics of macrodiversity reception in the presence of correlated Gamma long term fading and Rician short term fading are calculated and discussed in [8]. In the paper [9], macrodiversity system with macrodiversity SC receiver and two microdiversity SC receivers in the presence of small scale fading and large scale fading are considered and level crossing rate and average fade duration are calculated.

In this paper, macrodiversity system with macrodiversity SC receiver and two microdiversity MRC receivers in the presence of short term fading and long term fading are analysed. Received signal is subjected to $\eta-\mu$ multipath fading and Gamma shadowing. The level crossing rate of $\eta-\mu$ random process and level crossing rate of microdiversity MRC receivers output signal process are evaluated and these expressions are used for calculation level crossing rate of macrodiversity SC receiver output signal envelope. To the best knowledge of the authors, the second order statistics of macrodiversity system in the presence Gamma long term fading and $\eta-\mu$ long term fading is not reported in the available technical literature. 


\section{LEVEL CROSSING RATE OF $\eta-\mu$ RANDOM PROCESS}

Squared of $\eta-\mu$ random variable is:

$$
x^{2}=x_{1}^{2}+x_{2}^{2}+\cdots+x_{\mu}^{2}+x_{\mu+1}^{2}+\ldots+x_{2 \mu}^{2}
$$

where $x_{i}, i=1,2, \ldots, 2 m$ is Gaussian random processes with zero mean and independent. The first $\mu$ Gaussian random processes has variance $\sigma_{1}^{2}$ and the second $\mu$ Gaussian random processes has variance $\sigma_{2}^{2}$. The first derivate of $x$ is [9]:

$$
x \dot{x}=x_{1} \dot{x}_{1}+x_{2} \dot{x}_{2}+\cdots+x_{\mu} \dot{x}_{\mu}+x_{\mu+1} \dot{x}_{\mu+1}+\cdots+x_{2 \mu} \dot{x}_{2 \mu}
$$

The first derivate of Gaussian random process is Gaussian random process. The linear combination of Gaussian random processes is Gaussian random process. Therefore, $\dot{x}$ is Gaussian random process. Variance of $\dot{x}$ is:

$$
\sigma_{\dot{x}}^{2}=\frac{1}{x^{2}}\left(x_{1}^{2} \sigma_{\dot{x}_{1}}^{2}+x_{2}^{2} \sigma_{\dot{x}_{2}}^{2}+x_{\mu}^{2} \sigma_{\dot{x}_{\mu}}^{2}+x_{\mu+1}^{2} \sigma_{\dot{x}_{\mu+1}}^{2}+\ldots+x_{2 \mu}^{2} \sigma_{\dot{x}_{2 \mu}}^{2}\right)
$$

where is:

$$
\begin{aligned}
& \sigma_{\dot{x}_{1}}^{2}=\sigma_{\dot{x}_{2}}^{2}=\cdots \cdots=\sigma_{\dot{x}_{\mu}}^{2}=\pi^{2} f_{m}^{2} 2 \sigma_{1}^{2} \\
& \sigma_{\dot{x}_{\mu+1}}^{2}=\sigma_{\dot{x}_{\mu+2}}^{2} \cdots \cdots=\sigma_{\dot{x}_{2 \mu}}^{2}=\pi^{2} f_{m}^{2} 2 \sigma_{2}^{2}
\end{aligned}
$$

After substituting, the variance for $\dot{x}$ becomes:

where is:

$$
\begin{gathered}
\sigma_{\dot{x}}^{2}=\frac{2 \pi^{2} f_{m}^{2}}{x^{2}}\left(\sigma_{1}^{2}\left(x_{1}^{2}+x_{2}^{2}+\cdots+x_{\mu}^{2}\right)+\sigma_{2}^{2}\left(x_{\mu+1}^{2}+\ldots+x_{2 \mu}^{2}\right)\right)= \\
=\frac{2 \pi^{2} f_{m}^{2}}{x^{2}}\left(\sigma_{1}^{2} y_{1}+\sigma_{2}^{2} y_{2}\right)
\end{gathered}
$$

and

$$
\begin{gathered}
y_{1}=x_{1}^{2}+x_{2}^{2}+\cdots+x_{\mu}^{2} \\
y_{2}=x_{\mu+1}^{2}+x_{\mu+2}^{2} \cdots+x_{2 \mu}^{2}
\end{gathered}
$$

$$
\begin{aligned}
& x^{2}=y_{1}+y_{2} \\
& y_{1}=x^{2}-y_{2}
\end{aligned}
$$

Joint probability density function of $x, \dot{x}$ and $y_{2}$ is:

$$
\begin{aligned}
& p_{x \dot{x} y_{2}}\left(\begin{array}{lll}
x & \dot{x} & y_{2}
\end{array}\right)=p_{\dot{x}}\left(\dot{x} / x y_{2}\right) p_{x y_{2}}\left(x y_{2}\right)=p_{\dot{x}}\left(\dot{x} / x y_{2}\right) p_{y_{2}}\left(y_{2}\right) p_{x}\left(x / y_{2}\right) \\
& p_{x}\left(x / y_{2}\right)=\left|\frac{d y_{1}}{d x}\right| p_{y_{1}}\left(x^{2}-y_{2}\right)
\end{aligned}
$$

where is:

$$
\left|\frac{d y_{1}}{d x}\right|=2 x
$$


After substituting is:

$$
\begin{gathered}
p_{x}\left(x / y_{2}\right)=2 x p_{y_{1}}\left(x^{2}-y_{2}\right) \\
p_{x \dot{x} y_{2}}\left(x \dot{x} y_{2}\right)=2 x p_{y_{1}}\left(x^{2}-y_{2}\right) p_{y_{2}}\left(y_{2}\right) p_{\dot{x}}\left(\dot{x} / x y_{2}\right)
\end{gathered}
$$

Joint probability density function of $x$ and $\dot{x}$ is:

$$
p_{x \dot{x}}(x \dot{x})=\int_{0}^{x^{2}} d y_{2} p_{x \dot{x} y_{2}}\left(x \dot{x} y_{2}\right)=2 x \int_{0}^{x^{2}} d y_{2} p_{y_{1}}\left(x^{2}-y_{2}\right) p_{y_{2}}\left(y_{2}\right) p_{\dot{x}}\left(\dot{x} / x y_{2}\right)
$$

Level crossing rate of $x$ is:

$$
\begin{gathered}
N_{x}=\int_{0}^{\infty} d x \dot{x} p_{x \dot{x}}(x \dot{x})=2 x \int_{0}^{x^{2}} d y_{2} p_{y_{1}}\left(x^{2}-y_{2}\right) p_{y_{2}}\left(y_{2}\right) \int_{0}^{\infty} d \dot{x} \dot{x} p_{\dot{x}}\left(\dot{x} / x y_{2}\right)= \\
=2 x \int_{0}^{x^{2}} d y_{2} p_{y_{1}}\left(x^{2}-y_{2}\right) p_{y_{2}}\left(y_{2}\right) \frac{\sigma_{\dot{x}}}{\sqrt{2 \pi}}
\end{gathered}
$$

where is:

$$
\int_{0}^{\infty} \operatorname{dx} \dot{x} p_{\dot{x}}\left(\dot{x} / x y_{2}\right)=\frac{\sigma_{\dot{x}}}{\sqrt{2 \pi}}
$$

Random variables $y_{1}$ and $y_{2}$ are $\chi^{2}$ random variable:

$$
\begin{aligned}
& p_{y_{1}}\left(y_{1}\right)=\frac{2}{\Gamma(\mu / 2)}\left(\frac{1}{2 \sigma_{1}^{2}}\right)^{\mu / 2} y_{1}^{\mu-1} e^{-\frac{1}{2 \sigma_{1}^{2}} y_{1}^{2}}, \quad y_{1} \geq 0 \\
& p_{y_{2}}\left(y_{2}\right)=\frac{2}{\Gamma(\mu / 2)}\left(\frac{1}{2 \sigma_{2}^{2}}\right)^{\mu / 2} y_{2}^{\mu-1} e^{-\frac{1}{2 \sigma_{2}^{2}} y_{2}^{2}}, \quad y_{2} \geq 0
\end{aligned}
$$

After substituting, the level crossing rate of $x$ is:

$$
\begin{gathered}
N_{x}=\frac{2 \sqrt{2} \pi f_{m}}{\sqrt{2 \pi}}\left(\frac{2}{\Gamma(\mu / 2)}\right)^{2}\left(\frac{1}{2 \sigma_{1}^{2}}\right)^{\mu}\left(\frac{1}{2 \sigma_{2}^{2}}\right)^{\mu} \cdot \int_{0}^{x^{2}} d y_{2} \sqrt{\sigma_{1}^{2}\left(x^{2}-y_{2}\right)+\sigma_{2}^{2} y_{2}} . \\
\cdot\left(x^{2}-y_{2}\right)^{\mu-1} e^{-\frac{1}{2 \sigma_{1}^{2}}\left(x^{2}-y_{2}\right)^{2}} \cdot y_{2}^{\mu-1} e^{-\frac{1}{2 \sigma_{2}^{2}} y_{2}^{2}}, \quad y_{2} \geq 0
\end{gathered}
$$

\section{LEVEl CROSSING RATE OF MACRODIVERSITY OUTPUT SignAl ENVELOPE}

Macrodiversity system consisting of macrodiversity selection combining (SC) receiver and two microdiversity maximal ratio combining (MRC) receivers is considered. SC macrodiversity receiver is used to reduce long term fading effects on system 
performance and microdiversity MRC receivers are used to reduce short term fading effects on system performance. Signals envelopes at inputs of the first microdiversity MRC receiver are denoted with $x_{11}$ and $x_{12}$, signals envelope at inputs of the second microdiversity MRC receiver are denoted with $x_{21}$ and $x_{22}$. Signals envelopes at output of the first and the second microdiversity MRC receivers are denoted with $x_{1}$ and $x_{2}$, respectively. Signal at output of macrodiversity SC receiver is denoted with $x$. Model of considered system is shown on Figure 1.

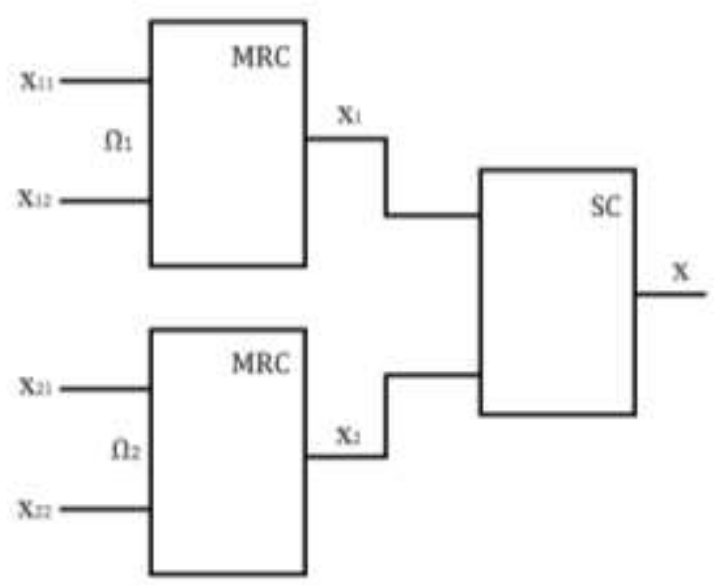

Fig. 1 Macrodiversity receiver with two microdiversity receivers.

Squared of $x_{1}$ and $x_{2}$ is:

$$
\begin{aligned}
& x_{1}^{2}=x_{11}^{2}+x_{12}^{2} \\
& x_{2}^{2}=x_{21}^{2}+x_{22}^{2}
\end{aligned}
$$

The level crossing rate of $x_{1}$ is:

$$
\begin{gathered}
N_{x_{1}}=\frac{2 \sqrt{2} \pi f_{m}}{\sqrt{2 \pi}}\left(\frac{2}{\Gamma(\mu)}\right)^{2}\left(\frac{1}{2 \sigma_{1}^{2}}\right)^{2 \mu}\left(\frac{1}{2 \sigma_{2}^{2}}\right)^{2 \mu} \int_{0}^{x^{2}} d y_{2} \sqrt{\sigma_{1}^{2}\left(x^{2}-y_{2}\right)+\sigma_{2}^{2} y_{2}} . \\
\cdot\left(x^{2}-y_{2}\right)^{2 \mu-1} y_{2}^{2 \mu-1} e^{-\frac{1}{2 \sigma_{1}^{2}}\left(x^{2}-y_{2}\right)^{2}-\frac{1}{2 \sigma_{2}^{2}} y_{2}^{2}}
\end{gathered}
$$

where is:

$$
\begin{gathered}
2 \sigma_{1}^{2}=\Omega_{1} ; \quad 2 \sigma_{2}^{2}=\Omega_{2} \\
\frac{\Omega_{1}}{\Omega_{2}}=q ; \quad \Omega_{1}=q \Omega_{2}
\end{gathered}
$$


After substituting level crossing rate of $x_{1}$ is:

$$
\begin{gathered}
N_{x_{1}}=\frac{2 \sqrt{2} \pi f_{m}}{\sqrt{2 \pi}}\left(\frac{2}{\Gamma(\mu)}\right)^{2} \frac{1}{q^{2 \mu} \Omega_{2}^{4 \mu-\frac{1}{2}}} \int_{0}^{x_{1}^{2}} d y_{2} \sqrt{q^{2}\left(x_{1}^{2}-y_{2}\right)+y_{2}} . \\
\cdot\left(x_{1}^{2}-y_{2}\right)^{2 \mu-1} y_{2}^{2 \mu-1} e^{-\frac{1}{\Omega_{2}}\left(\frac{1}{q^{2}}\left(x_{1}^{2}-y_{2}\right)^{2}+y_{2}^{2}\right)}
\end{gathered}
$$

The level crossing rate of the second microdiversity MRC receiver output signal process can be derived in the same way:

$$
\begin{gathered}
N_{x_{2}}=\sqrt{2} \pi f_{m}\left(\frac{2}{\Gamma(\mu)}\right)^{2} \frac{1}{q^{2 \mu} \Omega_{4}^{4 \mu-\frac{1}{2}}} \int_{0}^{x_{2}^{2}} d y_{4} \sqrt{q^{2}\left(x_{2}^{2}-y_{4}\right)+y_{4}} . \\
\cdot\left(x_{1}^{2}-y_{4}\right)^{2 \mu-1} y_{4}^{2 \mu-1} e^{-\frac{1}{\Omega_{4}}\left(\frac{1}{q^{2}}\left(x_{1}^{2}-y_{4}\right)^{2}+y_{4}^{2}\right)}
\end{gathered}
$$

Random variables $\Omega_{2}$ i $\Omega_{4}$ follow Gamma distribution:

$$
\begin{gathered}
p_{\Omega_{2} \Omega_{4}}\left(\Omega_{2} \Omega_{4}\right)=\frac{1}{\Gamma(\mathrm{c})\left(1-\rho^{2}\right) \rho^{c-1} \Omega_{0}^{\mathrm{c}+1}} \sum_{i_{1}=0}^{\infty}\left(\frac{\rho}{\Omega_{0}\left(1-\rho^{2}\right)}\right)^{2 i_{1}+c-1} . \\
\cdot \frac{1}{i_{1} ! \Gamma\left(i_{1}+c\right)} \Omega_{2}^{i_{1}+c-1} \Omega_{4}^{i_{1}+c-1} e^{-\frac{\Omega_{2}+\Omega_{4}}{\Omega_{0}\left(1-\rho^{2}\right)}}
\end{gathered}
$$

The level crossing rate of macrodiversity $\mathrm{SC}$ receiver output signal process is equal to level crossing rate of the first microdiversity MRC receiver output signal process when signal envelope average power at inputs of the first microdiversity receiver is higher of signal envelope average power at inputs of the second microdiversity MRC receiver. Level crossing rate of macrodiversity SC receiver output signal process is equal to level crossing rate of the second microdiversity MRC receiver output signal process when signal envelope average power at inputs of the second microdiversity MRC receiver is higher of signal envelope average power at inputs of the first microdiversity MRC receiver. Therefore, level crossing rate of macrodiversity SC receiver output signal process is:

$$
N_{x}=\int_{0}^{\infty} d \Omega_{2} \int_{0}^{\Omega_{2}} d \Omega_{4} N_{x_{1} / \Omega_{2}} p_{\Omega_{2} \Omega_{4}}\left(\Omega_{2} \Omega_{4}\right)+\int_{0}^{\infty} d \Omega_{4} \int_{0}^{\Omega_{2}} d \Omega_{2} N_{x_{2} / \Omega_{4}} p_{\Omega_{2} \Omega_{4}}\left(\Omega_{2} \Omega_{4}\right)=\mathrm{J}_{1}+\mathrm{J}_{2}
$$

The integral $\mathrm{J}_{1}$ is equal $\mathrm{J}_{2}$ : 


$$
\begin{aligned}
& \mathrm{J}_{1}=\mathrm{J}_{2}=\sqrt{2} \pi f_{m}\left(\frac{2}{\Gamma(\mu)}\right)^{2} \frac{1}{q^{2 \mu}} \frac{1}{\Gamma(\mathrm{c})\left(1-\rho^{2}\right) \rho^{\mathrm{c}-1} \Omega_{0}^{c+1}} \cdot \sum_{i_{1}=0}^{\infty}\left(\frac{\rho}{\Omega_{0}\left(1-\rho^{2}\right)}\right)^{2 i_{1}+\mathrm{c}-1} \\
& \cdot \frac{1}{i_{1} ! \Gamma\left(i_{1}+\mathrm{c}\right)} \cdot \int_{0}^{x_{2}^{2}} d y_{2} \sqrt{q\left(x_{2}^{2}-y_{2}\right)+y_{2}}\left(x_{2}^{2}-y_{2}\right)^{2 \mu-1} y_{2}^{2 \mu-1} \\
& \cdot \int_{0}^{\infty} d \Omega_{2} \Omega_{2}^{i_{1}+c-1-4 \mu+\frac{1}{2}} \cdot e^{-\frac{1}{\Omega_{4}}\left(\frac{1}{q}\left(x_{2}^{2}-y_{2}\right)^{2}+y_{2}^{2}\right)-\frac{\Omega_{2}}{\Omega_{0}\left(1-\rho^{2}\right)}} \\
& \cdot\left(\Omega_{0}\left(1-\rho^{2}\right)\right)^{i_{1}+c} \gamma\left(i_{1}+\mathrm{c}, \frac{\Omega_{2}}{\Omega_{0}\left(1-\rho^{2}\right)}\right) \\
& =\sqrt{2} \pi f_{m}\left(\frac{2}{\Gamma(\mu)}\right)^{2} \frac{1}{q^{2 \mu}} \frac{1}{\Gamma(\mathrm{c})\left(1-\rho^{2}\right) \rho^{\mathrm{c}-1} \Omega_{0}^{c+1}} \cdot \sum_{i_{1}=0}^{\infty}\left(\frac{\rho}{\Omega_{0}\left(1-\rho^{2}\right)}\right)^{2 i_{1}+\mathrm{c}-1} \\
& \cdot \frac{1}{i_{1} ! \Gamma\left(i_{1}+\mathrm{c}\right)} \cdot\left(\Omega_{0}\left(1-\rho^{2}\right)\right)^{i_{1}+\mathrm{c}} \frac{1}{i_{1}+\mathrm{c}} \frac{1}{\left(\Omega_{0}\left(1-\rho^{2}\right)\right)^{i_{1}+\mathrm{c}}} \\
& \cdot \sum_{j_{1}=0}^{\infty} \frac{1}{\left(i_{1}+\mathrm{c}+1\right)\left(j_{1}\right)} \frac{1}{\left(\Omega_{0}\left(1-\rho^{2}\right)\right)^{j_{1}}} \cdot \int_{0}^{x_{2}^{2}} d y_{2} \sqrt{q\left(x_{2}^{2}-y_{2}\right)+y_{2}}\left(x_{2}^{2}-y_{2}\right)^{2 \mu-1} y_{2}^{2 \mu-1} \\
& \cdot \int_{0}^{\infty} d \Omega_{2} \Omega_{2}^{2 i_{1}+2 \mathrm{c}-1-4 \mu+\frac{1}{2}+j_{1}} \cdot e^{-\frac{1}{\Omega_{4}}\left(\frac{1}{q}\left(x_{2}^{2}-y_{2}\right)^{2}+y_{2}^{2}\right)-\frac{2 \Omega_{2}}{\Omega_{0}\left(1-\rho^{2}\right)}}= \\
& =\sqrt{2} \pi f_{m}\left(\frac{2}{\Gamma(\mu)}\right)^{2} \frac{1}{q^{2 \mu}} \frac{1}{\Gamma(\mathrm{c})\left(1-\rho^{2}\right) \rho^{\mathrm{c}-1} \Omega_{0}^{c+1}} \cdot \sum_{i_{1}=0}^{\infty}\left(\frac{\rho}{\Omega_{0}\left(1-\rho^{2}\right)}\right)^{2 i_{1}+\mathrm{c}-1} \\
& \cdot \frac{1}{i_{1} ! \Gamma\left(i_{1}+\mathrm{c}\right)} \cdot \frac{1}{i_{1}+\mathrm{c}} \sum_{j_{1}=0}^{\infty} \frac{1}{\left(i_{1}+\mathrm{c}+1\right)\left(j_{1}\right)} \frac{1}{\left(\Omega_{0}\left(1-\rho^{2}\right)\right)^{j_{1}}} . \\
& \cdot \int_{0}^{x^{2}} d y_{2} \sqrt{q\left(x^{2}-y_{2}\right)+y_{2}}\left(x^{2}-y_{2}\right)^{2 \mu-1} y_{2}^{2 \mu-1} \\
& \cdot\left(\left(\frac{1}{2 q}\left(x^{2}-y_{2}\right)^{2}+y_{2}^{2}\right) \Omega_{0}\left(1-\rho^{2}\right)\right)^{i_{1}+\mathrm{c}-2 \mu+\frac{1}{4}+\frac{j_{1}}{2}} \\
& \cdot \mathrm{K}_{2 i_{1}+2 \mathrm{c}-4 \mu+\frac{1}{2}+j_{1}}\left(2 \sqrt{\frac{2}{\Omega_{0}\left(1-\rho^{2}\right)}}\left(\frac{1}{q}\left(x^{2}-y_{2}\right)^{2}+y_{2}^{2}\right)\right)
\end{aligned}
$$

where $K_{n}(x)$ is the modified Bessel function of the second kind, order $n$ and $\operatorname{argument} x$ [16].

\section{NUMERICAL RESULTS}

In Figure 2, level crossing rate of macrodiversity receiver output signal envelope process is presented for several values of Gamma long term fading severity parameters, Gamma long term fading correlation coefficient, the $\eta-\mu$ short term fading severity 
parameters and the $\eta-\mu$ short term fading $\eta$ parameter. For lower values SC receiver output signal, level crossing rate increases, reaches maximum and level crossing rate decreases for higher values SC receiver output signal. The influence of SC receiver output signal on level crossing rate is higher for lower values of SC receiver output signal. The level crossing rate decreases as Gamma long term fading severity parameter increases. The influence of Gamma long term fading severity parameters on level crossing rate is higher for lower values of Gamma long term fading severity parameter and lower values of SC receiver output signal envelope. The level crossing rate increases when the $\eta-\mu$ short term fading severity parameter decreases. The influence the $\eta-\mu$ short term fading parameter on level crossing rate is higher for lower values SC receiver output signal envelope lower values of Gamma long term fading severity parameter and lower values of Gamma long term correlation coefficient.

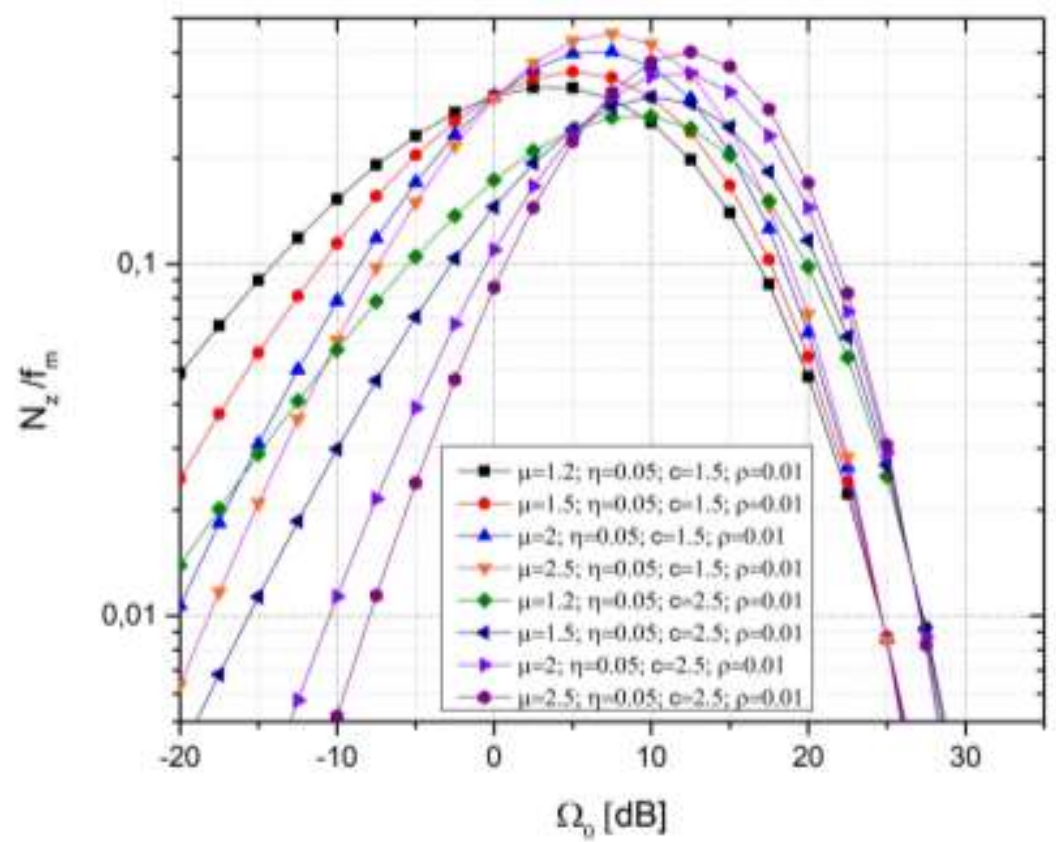

Fig. 2 Level crossing rate of macrodiversity SC receiver output signal envelope for different values of $\mu$ and $c$

The level crossing rate increases as Gamma long term fading correlation coefficient increases. The influence of Gamma long term fading correlation coefficient on level crossing rate is higher for lower values of Gamma long term fading severity parameters and lower values of the $\eta-\mu$ short term fading severity parameter. The level crossing rate increases when $\eta-\mu$ short term fading $\eta$ parameter decreases. The influence of $\eta-\mu$ short term fading $\eta$ parameter on level crossing rate is higher for lower values of Gamma long term fading severity parameter, lower values of Gamma long term fading correlation coefficient and lower values of $\eta-\mu$ short term fading severity parameters. The influence of $\eta-\mu$ short term fading $\eta$ parameter on level crossing rate is higher for lower values of $\mathrm{SC}$ receiver output signal envelope. 


\section{CONCLUSION}

In this paper, macrodiversity technique is used to reduce Gamma long term fading effects and $\eta-\mu$ short term fading effects on system performance. Macrodiversity SC receiver combines signal envelopes with antennas on base station distributed in cells resulting in system performance improvement. Microdiversity MRC receiver combines signal envelope with multiple antennas at base station resulting in short term fading effects reduction. Received signal experiences $\eta-\mu$ small scale fading and correlated Gamma large scale fading. The level crossing rate is important performance measure of wireless mobile communication system. In this paper, level crossing rate $\eta-\mu$ random process is calculated and this formulas can be used for evaluation average fade duration of wireless communication system operating over $\eta-\mu$ short term fading channel. The level crossing rate of MRC receiver output signal process is, also, calculated and average fade duration of wireless communication system with MRC operating over $\eta-\mu$ short term fading channel can be evaluated. These formulas are used for evaluation level crossing rate of macrodiversity SC receiver output signal process and this expression can be used for calculation average fade duration of macrodiversity in the presence $\eta-\mu$ short term fading and Gamma long term fading. Derived expression for level crossing rate of macrodiversity SC receiver output signal process can be used for evaluation level crossing rate of macrodiversity SC receiver output signal process in the presence Nakagami-m short term fading and Gamma long term fading. The influence of Gamma long term fading severity parameter, Gamma short term fading correlation coefficient, the $\eta-\mu$ short term fading severity parameter and the $\eta-\mu$ short term fading $\eta$ parameter on level crossing rate is analysed and studied.

\section{LITERATURE}

[1] M. D. Yacoub, The $\kappa-\mu$ distribution and the $\eta-\mu$ distribution. IEEE Antennas and Propag. Mag., vol. 49, pp. 68-81, Feb. 2007.

[2] W. C. Y. Lee, Mobile Communications Design Fundamentals. 2nd ed., John Wiley \& Sons, Inc, New York, 1993.

[3] Stüber GL. Principles of Mobile Communications. Kluwer Academic Publishers: Massachusetts, 1996.

[4] M. K. Simon, M. S. Alouini, Digital Communication over Fading Channels. John Wiley \& Sons, USA, 2000.

[5] Z. Hadzi-Velkov, "Level crossing rate and average fade duration of selection diversity with Rician-faded cochannel interferers," in IEEE Communications Letters, vol. 10, no. 9, pp. 649-651, Sept. 2006.

[6] S. Panic, M. Stefanovic, J. Anastasov, P. Spalevic, Fading and Interference Mitigation in Wireless Communications. CRC Press, USA, 2013.

[7] D. Stefanovic, S. Panic, P. Spalevic, "Second-order statistics of SC macrodiversity system operating over Gamma shadowed Nakagami-m fading channels," International Journal of Electronics and Communications, vol. 65, no. 5, pp. 413-418, 2011. [Online]. Available: http://dx.doi.org/10.1016/ j.aeue.2010.05.001

[8] N. Sekulovic, M. Stefanović, "Performance analysis of system with micro- and macrodiversity reception in correlated gamma shadowed Rician fading channels," Wireless Personal Communications, vol. 65, no. 1, pp. 143-156, 2012. [Online]. Available: http://dx.doi.org/10.1007/s11277-011-0232-8

[9] B. Jaksic, D. Stefanovic, M. Stefanovic, P. Spalevic, V. Milenkovic, "Level Crossing Rate of Macrodiversity System in the Presence of Multipath Fading and Shadowing," Radioengineering, vol. 24, no. 1, pp. 185-191, 2015. [Online]. Available: http://dx.doi.org/10.13164/re.2015.0185

[10] D. Krstić, M. Głabowski, D. Radenković and I. Temelkovski, Level crossing rate of MRC receiver over $\eta-\mu$ multipath fading. 9th International Symposium on Communication Systems, Networks \& Digital Sign (CSNDSP), Manchester, pp. 111-116, 2014. 
[11] A. D. Cvetkovic, M. Ĉ. Stefanovic, N. M. Sekulovic, D. N. Milic, D. M. Stefanovic, Z. J. Popovic, "Second-order statistics of dual SC macrodiversity system over channels affected by Nakagami-m fading and correlated gamma shadowing," Przeglad Elektrotechniczny, vol. 87, no. 6/2011, 2011.

[12] E. Mekic, N. Sekulovic, M. Bandjur, M. Stefanovic, P. Spalevic, "The distribution of ratio of random variable and product of two random variables and its application in performance analysis of multi-hop relaying communications over fading channels," Przeglad Elektrotechniczny, vol. 88, no. 7A, pp. 133-137, 2012.

[13] P. M. Shankar, "Analysis of microdiversity and dual channel macrodiversity in shadowed fading channels using a compound fading model," International Journal of Electronics and Communications (AEUE), vol. 62, no. 6, pp. 445-449, 2008. [Online]. Available: http://dx.doi.org/10.1016/j.aeue. 2007.06.008

[14] S. Mukherjee, D. Avidor, "Effect of microdiversity and correlated macrodiversity on outages in a cellular system," IEEE Transactions on Wireless Technology, vol. 2, no. 1, pp. 50-59, 2003. [Online]. Available: http://dx.doi.org/10.1109/TWC.2002.806363

[15] Marković, A., Perić, Z., Đošić, D., Smilić, M., \& Jakšić, B. (2015). Level crossing rate of macrodiversity system over composite Gamma shadowed alpha-kappa-mu multipath fading channel. Facta Universitatis, Series: Automatic Control And Robotics, 14(2), 99-109.

[16] S. Gradshteyn, I. M. Ryzhik, Table of Integrals, Series and Products. Academic Press, USA San Diego, 2000. 